\title{
Associação entre hábitos alimentares inadequados e inatividade física com fatores de risco cardiometabólicos: um estudo em Santa Cruz do Sul
}

\author{
Association between improper eating habits and physical \\ inactivity with cardiometabolic risk factors: a study in Santa Cruz \\ do Sul, Brazil
}

Miria Suzana Burgos', Luiza Silva de Oliveira, Luiza Pasa', Kelin Cristina Marques', Sonimar de Souza', Cláudia Daniela Barbian', Anelise Reis Gaya', Patrícia Molz², Silvia Isabel Rech Franke'

"Universidade de Santa Cruz do Sul (Unisc), Santa Cruz do Sul, RS, Brasil.

2Pontifícia Universidade Católica do Rio Grande do Sul (PUCRS), Porto Alegre, RS, Brasil

Recebido em: 20/01/2016 / Aceito em: 23/03/2016

mburgos@unisc.br

\section{RESUMO}

Objetivo: comparar os hábitos alimentares e de atividade física com fatores de risco cardiometabólicos de crianças e adolescentes. Método: trata-se de um estudo transversal, compreendendo uma amostra de 1963 escolares de ambos os sexos, com idade de 7 a 17 anos, pertencentes a 19 escolas da zona urbana e rural do município de Santa Cruz do Sul, RS. As questões referentes aos hábitos alimentares e prática de atividade física foram avaliadas por meio de questionário. Os fatores de risco cardiometabólicos avaliados foram: IMC, circunferência da cintura, percentual de gordura corporal, colesterol total e suas frações (HDL e LDL), triglicerídeos e glicose. Foi utilizado o programa SPSS 20.0 para a realização das análises estatísticas. Foram avaliados os valores médios, por meio da ANOVA, bem como o tamanho de efeito, considerando diferenças significativas para $p<0,05$. Resultados: observou-se que o consumo diário de 5 porções de frutas e verduras não apresentou associação com os fatores de risco cardiometabólicos. O hábito de evitar o consumo de alimentos gordurosos e doces, porém, apresentou um efeito de $0,3 \%(p=0,019)$ sobre o colesterol total e $0,5 \%(p=0,004)$ sobre o HDL. O consumo de 4 a 5 refeições variadas durante o dia apresentou efeito sobre o $\operatorname{IMC}(0,3 \% ; p=0,021)$ e sobre a circunferência da cintura $(0,5 \% ; p=0,004)$. A prática de atividade física apresentou efeito sobre o IMC $(0,3 \% ; p=0,017)$, glicose $(0,2 \% ; p=0,049)$ e $\operatorname{HDL}(0,4 \% ; p=0,011)$. Juntas, as questões relacionadas aos hábitos alimentares e à atividade física apresentaram um efeito significativo sobre o HDL $(2,1 \% ; p=0,001)$. Considerações finais: dessa maneira, conclui-se que foi encontrado um efeito pequeno, porém significativo, dos hábitos alimentares e da atividade física sobre o IMC e perfil lipídico, principalmente. Estudos futuros são necessários para a avaliação de outros componentes relacionados à obesidade e demais fatores de risco cardiometabólicos na população infanto-juvenil.

Palavras-chave: Obesidade; Crianças; Adolescentes; Hábitos alimentares; Exercício; Atividade motora.

\section{ABSTRACT}

Objective: compare eating and physical activity habits with cardiometabolic risk factors in children and teens. Method: this is a cross-sectional study, with 1963 boys and girls aged 7 to seventeen, belonging to 19 schools from rural and urban areas of Santa Cruz do Sul, Brazil. Eating habits and physical activity practice were evaluated through questionnaires. Cardiometabolic risk factors evaluated comprised: body mass index (BMI), waist circumference, body fat percentage, total cholesterol and fractions (HDL and $L D L)$, triglycerides and glucose. Statistical analyses were performed with software SPSS 20.0. Mean values were evaluated through ANOVA, as well as effect size, being significant when $p<0.05$. Results: we observe that daily consumption of 5 fruits and greens portions didn't present association with cardiometabolic risk factors. The habit of avoid consumption of fatty foods and sweets showed effect of $0.3 \%(p=0.019)$ on total cholesterol and $0.5 \%(p=0.004)$ on HDL. Consumption of 4 to 5 varied meals during the day presented effect on BMI 
$10.3 \% ; p=0.021$ ) and on waist circumference $10.5 \%$ $p=0.004)$. Physical activity habit showed effect on $B M I(0.3 \% ; p=0.017)$, glucose $(0.2 \% ; p=0.049)$ and $H D L(0.4 \% ; p=0.011)$. Together, questions related to food and physical activity habits presented a significant effect on $H D L 12.1 \% ; p=0.001)$. Closing remarks: we found a small effect, but significant, of eating and physical activity habits on BMI and lipid profile, mainly. Future surveys are necessary in order to evaluate of other factors related to obesity and cardiometabolic risk factors in children and teens.

Keywords: Obesity; Children; Teens; Eating habits; Physical activity; Motor activity.

\section{INTRODUCÃO}

A obesidade, assim como seu pré-estágio, o sobrepeso, pode ser considerada como uma concentração maior e anômala de gordura corporal, que pode ocasionar futuros problemas de saúde. ${ }^{1}$ Entre suas principais causas, está o desequilíbrio energético entre as calorias ingeridas e as utilizadas metabolicamente ${ }^{2-4}$ devido ao maior consumo de alimentos com alto teor energético (como os com grande concentração de gorduras) e a inatividade física. ${ }^{1}$ Desta forma, os atuais hábitos de vida, possivelmente podem explicar o crescimento da prevalência de sobrepeso e obesidade, sendo que, as mudanças neste episódio acarretariam diminuição da incidência dos casos, ocasionando modificações consequentes na prevalência. ${ }^{5}$

Essa transformação nos hábitos alimentares na sociedade atual acarreta em uma vida menos saudável, tanto na escolha errada dos alimentos, como nos altos níveis de sedentarismo da população, que tem como exemplo, o maior tempo assistindo televisão, desencadeando um desequilíbrio energético. ${ }^{6}$ Segundo a Organização Mundial da Saúde, isso pode ser resultado das modificações sociais e ambientais, vinculadas à deficiência de políticas nos departamentos de saúde, educação, indústria alimentícia, estratégias de mercado, meios de transporte e outros.

Desta forma, são imprescindíveis programas de educação que reforcem a primordialidade de hábitos alimentares saudáveis na infância e adolescência. ${ }^{7}$ Do mesmo modo, se faz necessário confrontar essa desorganização nutricional, pois a obesidade é uma condição de risco para o surgimento de doenças cardiovasculares $^{8,9}$ e da síndrome metabólica, ${ }^{8,10}$ assim como amplia as chances de crianças e adolescentes permanecerem obesos na fase adulta e serem acometidos por morte prematura. ${ }^{1}$

Segundo Damiani et al., ${ }^{11}$ as alterações cardiometabólicas e os riscos cardiovasculares são, sem dúvida, cada vez mais frequentes em crianças e adolescentes já em tenra idade, levando a apresentar problemas mais sérios futuramente. Um estudo feito em Minas Gerais, com 348 crianças, demonstrou que $24 \%$ delas estavam expostas ao risco cardiometabólico aumentado. ${ }^{12}$ Do mesmo modo, o crescimento da síndrome metabólica vem sendo observado em todo o mundo, tendo como fatores agravantes o padrão alimentar e a inatividade física. A síndrome metabólica é significante entre crianças e adolescentes, gerando um agravamento na presença da obesidade, quando podem-se observar alterações nos marcadores bioquímicos de riscos cardiovasculares. ${ }^{13,14}$

Diante do exposto, o presente estudo objetivou avaliar os hábitos alimentares e de atividade física e associá-los com fatores de risco cardiometabólicos de crianças e adolescentes do município de Santa Cruz do Sul, RS.

\section{MÉTODO}

O presente estudo, de caráter transversal, contou com amostra representativa de 1963 escolares de ambos os sexos, sendo 899 do sexo masculino e 1064 do sexo feminino, com idade de 7 a 17 anos, pertencentes a 19 escolas da zona urbana e rural do município de Santa Cruz do Sul, RS. A pesquisa foi previamente encaminhada ao Comitê de Ética em Pesquisa da Unisc, sendo aprovada sob o protocolo número 2959/2011. Encaminhou-se o Termo de Consentimento Livre e Esclarecido (TCLE) aos pais ou responsáveis dos escolares, contendo informações importantes da aplicação da pesquisa e, após sua autorização, iniciou-se a coleta de dados.

As questões referentes aos hábitos alimentares e a prática de atividade física foram avaliadas por meio de questionário adaptado de Nahas, Barros e Francalacci. ${ }^{15}$ Para a avaliação dos hábitos alimentares, foram consideradas as seguintes questões: 1) sua alimentação diária inclui pelo menos 5 porções de frutas e verduras?; 2) você evita ingerir alimentos gordurosos e doces? e 3) você faz 4 a 5 refeições variadas durante o dia? Os escolares foram questionados também se praticavam, pelo menos, 30 minutos de atividades moderadas/ intensas, de forma contínua, por pelo menos 5 vezes por semana.

Para avaliação dos fatores de risco cardiometabólicos, foram avaliados o índice de massa corporal (IMC), a circunferência da cintura (CC), perfil lipídico (triglicerídeos, colesterol total e frações - HDL-c e LDL-c) e glicose. O IMC foi calculado pela razão do peso $(\mathrm{kg})$ pela altura $^{2}(\mathrm{~m})$; a CC foi avaliada com fita métrica inelástica com resolução de $1 \mathrm{~mm}$, sendo utilizada como referência a parte mais estreita do tronco, entre as costelas e a crista ilíaca. Os indicadores bioquímicos (glicose, triglicerídeos, colesterol total e frações HDL-c e LDL-c) foram avaliados em amostras de soro dos escolares que estavam em jejum de 12 horas. Foram utilizados kits comerciais DiaSys (DiaSys Diagnostic Systems, Alemanha), no equipamento Miura One (I.S.E., Roma, Itália). O colesterol LDL foi calculado pela equação de Friedewald, Levy e Fredrickson. ${ }^{16}$

Foi utilizado o programa SPSS 20.0 para a realização das análises estatísticas. Foram avaliados os valores médios, por meio da ANOVA, bem como o tamanho de efeito, considerando diferenças significativas para $p<0,05$.

\section{RESULTADOS E DISCUSSÃO}

De acordo com os dados descritivos demonstrados na tabela 1, observou-se que, para os hábitos alimentares, não houve diferença entre os sexos. Os dados obtidos mostraram-se preocupantes, uma vez que a maior parte nunca ou somente às vezes consumiam 
5 porções de frutas e verduras por dia $(75,4 \%$ entre os meninos e $77,3 \%$ entre as meninas) (Tabela 1). Em um estudo transversal realizado por Rocha et al., ${ }^{17} \mathrm{com}$ adolescentes portugueses, foi verificado que $61 \%$ dos mesmos consumiam diariamente saladas ou legumes e $76 \%$ deles consumiam frutas, similar ao resultado do presente estudo. O mesmo estudo também mostrou que $13 \%$ e $6 \%$ dos adolescentes respectivamente, relataram ingerir saladas ou legumes e frutas menos do que uma vez por semana. Outro estudo retrospectivo, avaliando os hábitos alimentares de 616 crianças da cidade de Pelotas-RS, relataram que entre os escolares avaliados, foi encontrado um consumo de apenas $2,11 \%$ de legumes e frutas diariamente, valor muito inferior ao encontrado no presente estudo. ${ }^{18}$

No nosso estudo, houve um percentual elevado de escolares que não realizavam 4 a 5 refeições variadas durante o dia $(48,6 \%$ e $46,9 \%$ para meninos e meninas, respectivamente) (Tabela 1). Diferentemente, o estudo de Rocha et al. ${ }^{17}$ observaram que a maioria dos adolescentes $(77 \%)$ realizavam 5 ou mais refeições diárias e $17 \%$ relataram realizar quatro refeições. Entretanto, Dutra et al. ${ }^{18}$ verificaram um maior consumo de refeições variadas $(75,2 \%$ das crianças avaliadas, realizavam pelo menos três refeições e dois lanches por dia) que o presente estudo.

O consumo elevado de alimentos de alto teor energético, principalmente com grandes concentrações de gordura e de açúcar, está dentre os principais fatores de risco para a obesidade infantil. ${ }^{19}$ No presente estudo, a maioria dos meninos $(72,5 \%)$ e das meninas $(71,6 \%)$ consumiam alimentos gordurosos e doces. Em um estudo realizado numa região da Espanha com 3061 crianças, também foi observado um consumo elevado de alimentos associados ao risco de obesidade. ${ }^{20}$

Com relação à prática de atividade física (Tabela 1), nosso estudo mostrou que os meninos eram mais ativos $(p<0,001)$, sendo que $54,9 \%$ praticavam pelo menos 30 minutos de atividade física moderada/intensa, de forma contínua ou acumulada, cinco ou mais dias durante a semana. Já, o percentual de escolares que não praticava atividade física, ou praticava somente às vezes, foi elevado em ambos os sexos no presente estudo $(45,1 \%$ entre os meninos e $58,5 \%$ entre as meninas $)$. Corroborando com nosso estudo, Venâncio, Teixeira e Silva, ${ }^{21}$ num estudo de caráter epidemiológico na cidade de Anápolis-GO, avaliando 1982 crianças de 7 a 9 anos, verificaram que os meninos eram significativamente $(p<0,000)$ mais ativos que as meninas.

Farias Júnior et al., ${ }^{22}$ em estudo transversal composto por 2.874 estudantes de 14 a 19 anos de idade em João Pessoa-PB, também verificaram que o sexo masculino se mostrou fisicamente mais ativo $(p<0,001)$. Eles ainda constataram que o percentual de jovens que não praticavam atividade física (conforme as recomendações) foi elevado, principalmente no sexo feminino e nos escolares pertencentes a famílias de menor nível socioeconômico. Entre os escolares mais propensos a serem ativos, estavam os que tinham uma percepção positiva do seu estado de saúde e os que participavam das aulas de Educação Física. O estudo de Santiago et al. ${ }^{20}$ também relataram que a inatividade física foi mais prevalente entre as meninas, levando a um aumento do risco cardiometabólico.

De acordo com a Tabela 2, observou-se que o consumo diário de 5 porções de frutas e verduras não apresentou associação com os fatores de risco cardiometabólicos. O hábito de evitar o consumo de alimentos gordurosos e doces, porém, apresentou um efeito de $0,3 \%(p=0,019)$ sobre o colesterol total e $0,5 \%$ $(p=0,004)$ sobre o HDL-c. Além disso, o consumo de 4 a 5 refeições variadas durante o dia apresentou efeito sobre o IMC $(0,3 \% ; p=0,021)$ e sobre a CC $10,5 \%$; $p=0,004)$. Diferentemente do nosso estudo, Ribeiro et al., ${ }^{23}$ comparando indivíduos com hábitos alimentares não saudáveis (que consumiam alimentos ricos em gordura e muito pobres em vegetais, frutas e fibras) e indivíduos com hábitos alimentares saudáveis, observaram que os indivíduos não apresentaram diferenças significativas em relação a níveis baixos de HDL-c, e níveis elevados de colesterol total e LDL-C.

Em outro estudo transversal, realizado com 2481 escolares com idade entre 11 a 14 anos, de escolas da rede pública e privada de Florianópolis-SC, o consumo menor de três refeições diárias e a ingestão de alimentos de risco, mais de três dias da semana, associou-se com excesso de gordura corporal, porém, não houve associação com atividade física. ${ }^{24}$ Semelhantemente, em estudo transversal realizado com 906 adolescentes de 14 a 19 anos, o consumo de café da manhã, alimentos rápidos, bolos e doces foi significativamente associada com sobrepeso e obesidade $(p<0,05) \cdot{ }^{25}$

No presente estudo, a prática de atividade física apresentou efeito significativo sobre o IMC 10,3\%;

Tabela 1 - Perfil dos hábitos alimentares e de atividade física dos escolares.

\begin{tabular}{|c|c|c|c|}
\hline & $\begin{array}{c}\text { Masculino } \\
\text { n (\%) }\end{array}$ & $\begin{array}{c}\text { Feminino } \\
\mathrm{n}(\%)\end{array}$ & $\mathbf{p}$ \\
\hline Consumo de 5 porções de frutas e verduras por dia & & & 0,170 \\
\hline Nunca/às vezes & $678(75,4)$ & $823(77,3)$ & \\
\hline Sempre/quase sempre & $221(24,6)$ & $241(22,7)$ & \\
\hline Evita consumir alimentos gordurosos e doces & & & 0,346 \\
\hline Nunca/às vezes & $652(72,5)$ & $762(71,6)$ & \\
\hline Sempre/quase sempre & $247(27,5)$ & $302(28,4)$ & \\
\hline Faz 4 a 5 refeições variadas durante o dia & & & 0,239 \\
\hline Nunca/às vezes & $437(48,6)$ & $499(46,9)$ & \\
\hline Sempre/quase sempre & $462(51,4)$ & $565(53,1)$ & \\
\hline Atividade física* & & & $<0,001$ \\
\hline Nunca/às vezes & $405(45,1)$ & $622(58,5)$ & \\
\hline Sempre/quase sempre & $494(54,9)$ & $442(41,5)$ & \\
\hline
\end{tabular}

*ao menos 30 minutos de atividade física moderada/intensa, de forma contínua ou acumulada, 5 ou mais dias durante a semana. 
Tabela 2 - Efeito dos hábitos alimentares e da atividade física sobre os fatores de risco cardiometabólicos.

\begin{tabular}{|c|c|c|}
\hline & $\begin{array}{l}\text { Tamanho de } \\
\text { efeito (\%) }\end{array}$ & p \\
\hline \multicolumn{3}{|c|}{$\begin{array}{l}\text { Consumo de } 5 \text { porções de frutas e } \\
\text { verduras por dia }\end{array}$} \\
\hline IMC & 0 & 0,910 \\
\hline Circunferência da cintura & 0 & 0,560 \\
\hline Glicose & 0,1 & 0,189 \\
\hline Colesterol total & 0,1 & 0,255 \\
\hline Colesterol HDL & 0 & 0,082 \\
\hline Colesterol LDL & 0,2 & 0,827 \\
\hline Triglicerídeos & 0 & 0,456 \\
\hline \multicolumn{3}{|c|}{$\begin{array}{l}\text { Evita consumir alimentos gordurosos } \\
\text { e doces }\end{array}$} \\
\hline IMC & 0,1 & 0,241 \\
\hline Circunferência da cintura & 0,1 & 0,272 \\
\hline Glicose & 0 & 0,882 \\
\hline Colesterol total & 0,3 & 0,019 \\
\hline Colesterol HDL & 0,5 & 0,004 \\
\hline Colesterol LDL & 0,5 & 0,700 \\
\hline Triglicerídeos & 0 & 0,483 \\
\hline \multicolumn{3}{|c|}{ Faz 4 a 5 refeições variadas durante o dia } \\
\hline IMC & 0,3 & 0,021 \\
\hline Circunferência da cintura & 0,5 & 0,004 \\
\hline Glicose & 0,1 & 0,180 \\
\hline Colesterol total & 0 & 0,776 \\
\hline Colesterol HDL & 0 & 0,911 \\
\hline Colesterol LDL & 0 & 0,496 \\
\hline Triglicerídeos & 0 & 0,976 \\
\hline \multicolumn{3}{|l|}{ Atividade Física } \\
\hline IMC & 0,3 & 0,017 \\
\hline Circunferência da cintura & 0,2 & 0,064 \\
\hline Glicose & 0,2 & 0,049 \\
\hline Colesterol total & 0,1 & 0,323 \\
\hline Colesterol HDL & 0,1 & 0,011 \\
\hline Colesterol LDL & 0,4 & 0,772 \\
\hline Triglicerídeos & 0 & 0,289 \\
\hline
\end{tabular}

Hábitos alimentares e atividade

física*

$\begin{array}{lll}\text { IMC } & 1,1 & 0,153 \\ \text { Circunferência da cintura } & 1,2 & 0,115 \\ \text { Glicose } & 1,1 & 0,144 \\ \text { Colesterol total } & 0,9 & 0,349 \\ \text { Colesterol HDL } & 2,1 & 0,001 \\ \text { Colesterol LDL } & 0,7 & 0,583 \\ \text { Triglicerídeos } & 0,9 & 0,324\end{array}$

*Todas as questões referentes aos hábitos alimentares e prática de atividade física, em um mesmo modelo.

$p=0,017)$, sobre a glicemia $(0,2 \% ; p=0,049)$ e sobre a HDL-c $(0,4 \% ; p=0,011)$. Juntas, as questões relacionadas aos hábitos alimentares e à atividade física apresentaram um efeito significativo apenas sobre o HDL-c $(2,1 \% ; p=0,001)$. Um estudo transversal realizado no México, com 1309 crianças e adolescentes, com idades entre 5 e 17 anos, de ambos os sexos, objetivou analisar a relação do IMC, situação nutricional e atividade física leve com riscos cardiometabólicos, sendo observado que a atividade física leve associou-se ao risco cardiometabólico. ${ }^{26}$

Al-Haifi et al. ${ }^{25}$ identificaram em um total de 906 adolescentes árabes de ambos os sexos, com idade entre 14 e 19 anos, que entre os meninos, as atividades físicas moderadas e vigorosas foram negativamente associadas com sobrepeso e obesidade $(p<0,05)$. Também, a inatividade física demonstrou ser diretamente associada ao padrão alimentar, bem como à CC, ao peso e obesidade abdominal, significativamente, num estudo realizado na China por Adair et al. ${ }^{27}$ (2014), no qual participaram 9.000 crianças e adolescentes.

No presente estudo, a prática de atividade física apresentou efeito sobre a glicemia e HDL-c, e as questões relacionadas à combinação de hábitos alimentares e atividade física apresentaram um efeito significativo sobre o HDL-c. O estudo de Campos et al. ${ }^{28}$ de caráter transversal, com 490 escolares com idades entre 10 e 18 anos verificou que os níveis de atividade física dos adolescentes do sexo masculino sedentários apresentaram mais de 4 vezes maiores chances $(p=0,003)$ de serem hipercolesterolêmicos, quando comparados aos escolares mais ativos. No sexo feminino não foram encontradas associações significativas. Em um estudo transversal realizado em Salvador-BA, com 937 crianças e adolescentes com idade entre 7 e 14 anos, de ambos os sexos, a dislipidemia foi associada ao baixo consumo de alimentos protetores, ao consumo moderado e alto de alimentos de risco e ao sobrepeso e obesidade. Do mesmo modo, foi observado em $67,5 \%$ dos escolares, consumo de alimentos de moderado e alto risco para as dislipidemias. ${ }^{29}$

Assim como no nosso estudo, Bergmann et al. ${ }^{30}$ em estudo transversal de base escolar com 1.294 escolares de 7 a 12 anos, de Caxias do Sul-RS, não encontrou associações entre hábitos alimentares e colesterol total. O autor ressalta que a associação de um estilo de vida ativo com hábitos alimentares adequados, podem ser uma grande ferramenta na diminuição dos níveis de colesterol.

\section{CONCLUSÃO}

A partir dos dados encontrados, conclui-se que existe um efeito pequeno, porém significativo, dos hábitos alimentares e da atividade física sobre os fatores de risco cardiometabólicos de crianças e adolescentes, principalmente sobre o perfil lipídico. Desta forma, torna-se necessário reforçar a importância de estudos futuros para a avaliação de outros componentes relacionados aos fatores de risco cardiometabólicos na população infanto-juvenil, assim como evidenciar a necessidade de ações voltadas ao incentivo de hábitos alimentares saudáveis e da prática de atividades físicas satisfatórias, para que desta forma consiga-se alcançar melhoras na saúde e qualidade de vida dessa população.

\section{REFERÊNCIAS}

1. WHO. World Health Organization. Obesity and overweight, 2013. Disponível em: http://www.who.int/mediacentre/ factsheets/fs311/en/index.html. Acesso em: 27 de março/2016.

2. Astrup A, Brand-Miller J. Diet composition and obesity. The Lancet 2012;379(9821):1100.

3. Veldhuis L, Vogel I, Renders CM, Rossem LV, Oenema A, Hirasing RA, Raat H. Behavior risk factors for overweight 
in early child- hood: The Be active, eat right study. International Journal of Behavioral Nutrition and Physical Activity 2012;9:74-81.

4. Hills AP, Andersen LB, Byrne NM. Physical activity and obesity in children. British Journal of Sports Medicine 2011; 45(11):866-870.

5. Coelho LG, Cândido APC, Machado-Coelho GLL, Freitas SN. Association between nutritional status, food habits and physical activity level in schoolchildren. J Pediatri 2012; 88:406-412.

6. De Craemer M, De Decker E, De Bourdeaudhuij L, Vereecken C, Deforche B, Manios Y, Cardon G. Correlates of energy balance-related behaviours in preschool children: a systematic review. Obes Rev 2012;13(1):13-28.

7. Grosso G, Galvano F. Mediterranean diet adherence in children and adolescents in southern European countries. NFS Journal 2016; 3:13-19.

8. Di Renzo L, Tyndall E, Gualtieri P, Carboni C, Valente R, Ciani AS, Tonini MG, De Lorenzo A. Association of body composition and eating behavior in the normal weight obese syndrome. Eat Weight Disord 2016;21:99-106.

9. Fernandes RA, Rosa CSC, Silva CB, Bueno DR, Oliveira AR, Freitas Júnior IF. Desempenho de diferentes valores críticos de índice de massa corporal na identificação de excesso de gordura corporal e obesidade abdominal em adolescentes. Rev Assoc Med Bras 2007;3:515-519.

10. Costa RF, Santos NS, Goldraich NP, Barski TF, Andrade KS, Kruel LFM. Metabolic syndrome in obese adolescents: a compar-ison of three different diagnostic criteria. J Pediatri 2012; 88:303-309.

11. Damiani $D$, Kuba VM, Cominato L, Damiani D, Dichrchekenian V, Menezes Filho HC. Síndrome metabólica em crianças e adolescentes: dúvidas na terminologia, mas não nos riscos cardiometabólicos. Arq Bras Endocrinol Metab 2011; 55(8):576-582.

12. Villa JKD, Silva AR, Santos TSS, Ribeiro AQ, Sant'ana LFR Metabolic syndrome risk assessment in children: use of a single score. Rev Paul Pediatr 2015;33(2):187-193.

13. Caranti DA, Lazzer S, Dâmaso AR, Agosti F, Zennaro $R$, Mello MT, Tufik S, Sartorio A. Prevalence and risk factors of metabolic syndrome in Brazilian and Italian obese adolescents: a comparison study. Int J Clin Pract 2008; 62(10):1526-1532.

14. Weiss R, Dziura J, Burgert TS, Tamborlane WV, Taksali SE, Yeckel CW, Allen K, Lopes M, Savove M, Morrison J, Sherwin RS, Caprio S. Obesity and the metabolic syndrome in children and adolescents. N Engl J Med 2004;350(23):2362-2374.

15. Nahas MV, Barros MVG, Francalacci VL. O Pentáculo do Bem-Estar. Base conceitual para avaliação do estilo de vida de indivíduos ou grupos. Rev Bras Ativ Fís Saúde 2000; 5(2):48-59.

16. Friedewald WT, Levy RI, Fredrickson DS. Estimation of the concentration of low-density lipoprotein cholesterol in plasma, without use of the preparative ultracentrifuge. Clin Chem 1972;18(6):499-502.
17. Rocha T, Rocha E, Alves AC, Medeiros AM, Francisco V, Silva S, Mendes GI, Rato Q, Bourbon M. Cardiovascular risk profile of righ school students: a cross-sectional study. Rev Port Cardiol 2014;33(9):525-534.

18. Dutra GF, Kaufmann CC, Pretto ADB, Albernaz EP. Sedentary lifestyle and poor eating habits in childhood:a cohort study. Ciênc Saúde Coletiva 2016;21(4):1051-1059.

19. Vio F, Fretes G, Montenegro E, González CG, Salinas J. Prevention of Children Obesity: A Nutrition Education Intervention Model on Dietary Habits in Basic Schools in Chile. Food Nutri Sci 2015;6(13):1221.

20. Santiago S, Cuevo M, Zazpe I, Ortega A, Garcia-Perea A, Martinez JA. Situación ponderal, hábitos alimentarios y deportivos en población castellano-manchega de 6 a 12 años. An Pediatrí. 2014; 80(2):89-97.

21. Venâncio PEM, Teixeira CGO, Silva FMS. Excesso de peso, nível de atividade física e hábitos alimentares em escolares da cidade de Anápolis-GO. Rev Bras Ciên Esporte 2013;35(2):441-453.

22. Farias Júnior JC, Lopes AS, Mota J, Hallal PC. Physical activity practice and associated factors in adolescents in Northeastern Brazil. Rev Saúde Pública 2012;46(3).

23. Ribeiro ROC, Lotufo, PA, Lamounier JA, Oliveira RG, Soares JF, Botter DA. Additional Cardiovascular Risk Factors Associated with Excess Weigth in Children and Adolescents. The Belo Horizonte Heart Study. Arq Bras Cardiol 2006; 86(6).

24. D'avila GL, Silva DAS, Vasconcelos FAG. Associação entre consumo alimentar, atividade física, fatores socioeconômicos e percentual de gordura corporal em escolares. Ciênc Saúde Coletiva 2016;21(4):1071-1081.

25. Al-haifi AR, Al-fayez MA, Al-athari BI, Al-ajmi FA, Allafi AR, Al-hazzaa HM, Musaiger AO. Relative contribution of physical activity, sedentary behaviors, and dietary habits to the prevalence of obesity among Kuwaiti adolescents. Food Nutri Bulletin 2013;34(1):6-13.

26. Cárdenas LMC, Garcia AIB, Velasco BIE, Islas CL, Romeroc JP, Cruzc M, Portillo MG. Leisure-time physical activity and cardiometabolic risk among children and adolescents. J Pediatr 2015;91(2):136-142.

27. Adair LS, Gordon-Larsen P, Du SF, Zhang B, Popkin BM. The emergence of cardiometabolic disease risk in Chinese children and adults: consequences of changes in diet, physical activity and obesity. Obes Rev 2014;15(1):49-59.

28. Campos W, Stabelini Neto A, Bozza R, Ulbrich AZ, Bertin RL, Mascarenhas, LPG, Silva SG, Sasaki JE. Actividad física, consumo de lípidos y factores de riesgo para aterosclerosis em adolescentes. Arq Bras Cardiol 2010;94(5):583-589.

29. Alcântara neto OD, Silva RC, Assis AM, Pinto EJ. Factors associated with dyslipidemia in children and adolescents enrolled in public schools of Salvador, Bahia. Rev Bras Epidemiologia 2012;15(2):335-345.

30. Bergmann MLA, Bergmann GG, Halpern R, Rech RR, Constanzi CB, Alli LR. Associated Factors to Total Cholesterol: School Based Study in Southern Brazil. Arq Bras Cardiol 2011;97(1). 